\title{
SARCOPTIDES PLUMICOLES DES OISEAUX D'INDOCHINE
}

\author{
Par Jean GAUD et M.-L. PETITOT
}

Les sarcoptidés plumicoles (Analgesidæ) vivent en parasites sur les plumes des oiseaux (1). Ils semblent se nourrir, non des plumes elles-mêmes, mais des sécrétions qui recouvrent ces dernières ou, peut-être, des êtres inférieurs vivant dans ces sécrétions. Ces acariens ont fait l'objet de bonnes études morphologiques et systématiques. Leur biologie est moins bien connue. Elle pose d'intéressants problèmes : répartition selon les hôtes, les pays et les saisons, cycles biologiques, spécificité parasitaire, rôle vecteur éventuel pour d'autres parasites aviaires, etc...

Au cours de l'année 1946, nous avons eu l'occasion d'examiner un petit nombre d'oiseaux sauvages de Cochinchine, Cambodge et SudAnnam. Nous avons recherché systématiquement chez eux les sarcoptidés plumicoles, avec l'idée que ces acariens pouvaient jouer un rôle dans la transmission de certains cestodes. Nos recherches dans ce sens ont été négatives, mais elles ont été l'occasion d'identifier plusieurs espèces de sarcoptidés nouvelles pour l'Indochine. Etant donnée l'indigence des connaissances à ce sujet, nous pensons intéressant de publier un catalogue de nos trouvailles.

E.-F. Houdemer, dans ses Recherches de parasitologie comparée indochinoise (1938), consacre aux acariens plumicoles un paragraphe d'une telle brièveté qu'il peut être reproduit in extenso :

"La plupart des oiseaux domestiques ou sauvages du Tonkin en sont infestés. Il serait intéressant d'en faire une étude systématique qui réserverait certainement des trouvailles. Nous mentionnerons simplement que, chez trois espèces d'oiseaux : le pigeon domestique, Streptopelia orientalis orientalis Latham et Egretta garzetta garzetta L., nous avons vu des Hypodectes (nymphes de Falculifer) formant de vastes colonies dans le tissu conjonctif sous-cutané. »

Il est exact que nos connaissances sur les sarcoptidés plumicoles de l'Indochine sont pauvres. Elles le sont moins, toutefois, que ne le

(1) Plus rarement à l'intérieur du tuyau des plumes.

ANn. de Parasitologie, T. X́XIII, xos 5-6, - 1948, p. 337-347. 
laisse croire Houdemer. Cinq espèces d'Analgesidæ avaient été décrites par Trouessart (1885 et 1886), d'oiseaux originaires d'Indochine.

Megninia ibidis Tr. sur Phasianus torquatus d'Annam.

Freyana leclerci Tr. sur Lobivanellus indicus atrinuchalis de Cocninchine.

Pseudalloptes calcaratus Mégnin et Tr. et Psoroptoides psoroptopus $\mathrm{Tr}$. sur Dichoceros bicornis de Cochinchine.

Psoroptoides psoroptopus Tr. sur Dichoceros bicornis de Cochinchine.

Pterolichus phylloproctus Mégnin et Tr. sur Haliætus leucogaster d'Indochine.

D'autre part, plusieurs espèces de sarcoptidés plumicoles ont été identifiées dans des pays voisins de l'Indochine, sur des espèces aviaires faisant partie de la faune indochinoise. Ces sarcoptidés seront vraisemblablement tous retrouvés en Indochine. Nous donnons, à la fin de notre travail, un tableau de ces espèces.

Enfin, dans la collection du Muséum d'histoire naturelle de Paris (1), nous avons pu retrouver plusieurs espèces récoltées sur des oiseaux d'Indochine, identifiées ou classées et dénommées par Trouessart, mais qui n'avaient, à notre connaissance, fait l'objet d'aucune publication. Nous joignons ces espèces au catalogue de nos propres trouvailles.

Pterolichinæ, - Dans cette sous-famille, les pattes antérieures sont inermes dans les deux sexes. L'abdomen de la femelle est entier, recouvert dorsalement par une plaque notogastrique.

Genre Pseudalloptes (les pattes de la IV ${ }^{\circ}$ paire plus développées chez le mâle qu'elles ne le sont chez la femelle). Un Pseudalloptes sp.? figure dans la collection Trouessart, récolté sur l'Eurylaime Cymbirhynchus macrorhynchus de Cochinchine.

Genre Pterolichus (pattes subégales dans les deux sexes).

$P$. delibatus Robin et Megnin. Sur trois corbeaux Corvus macrorhynchus Wagler, tués à Ban Me Thuot, nous avons trouvé en grande abondance cette espèce, parasite cosmopolite des corvidés.

$P$. obtusus Robin, cosmopolite également, parasite des galliformes, figure dans la collection Trouessart, récolté sur le faisan prélat Diardigallus diardi de Cochinchine.

Dans la collection du Muséum également, nous avons retrouvé :

(1) Mise à notre disposition par le $\mathrm{D}^{\mathrm{r}}$ Mare André que nous remercions ici de son amabilité et de son aide compétente. 
P. rehbergi Canestrini et Berlese, provenant d'un vanneau (lobivanellus indicus) de Cochinchine.

$P$. vexillarius Mégnin et Trouessart, récolté sur divers calaos de Cochinchine.

Genre Pteronyssus (les pattes de la $\mathrm{III}^{\circ}$ paire, hypertrophiées chez le mâle).

$P$. lagopus Trouessart, espèce dont, à notre connaissance, la description n'a pas été publiée, figure dans la collection Trouessart, récolté sur Garrulax bellangeri de Cochinchine.

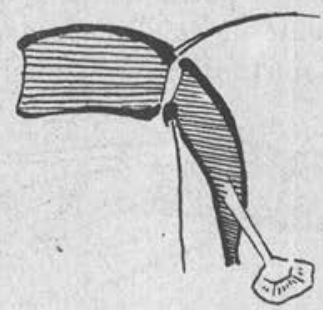

Fig. 1. - Extrémité d'une patte de la $\mathrm{III}^{\circ}$ paire chez Pteronyssus lonchure of.

$P$. lonchuræ Sugimoto. C'est à cette espèce récemment décrite que nous croyons devoir rapporter les acariens trouvés en Cochinchine sur Munia (=lonchura) punctatula topela Swinhoe et sur Ploceella chrysea (Hume). Les ambulacres sont remarquables par la raideur de leur tige (fig. 1).

$P$. truncatus Trouessart a été trouvé en grande abondance chez plusieurs martins : Acridotheres tristis L. et Gracupica leucocephala Giglioli et Salvadori, du Cambodge et du Sud-Annam (pl. I $a$ et $b$ ).

Nous avons enfin trouvé de très rares exemplaires de Pteronyssus, d'identification difficile, sur Passer flaveolus, Iole olivacea, Anthus richardi.

Analgesinæ, - Cette sous-famille est caractérisée par le grand développement d'une ou des deux paires de pattes postérieures chez certains individus mâles dits hétéromorphes. Les pattes antérieures sont armées dans les deux sexes, ornées de tubercules olécraniens et de manchettes. L'abdomen des femelles est entier. La plaque notogastrique fait généralement défaut chez elles.

Genre Analges (la troisième paire de pattes, très hypertrophiée chez le mâle hétéromorphe, ne porte pas d'ambulacres à son extrémité. 
A. humulus Trouessart, espèce dont, à notre connaissance, là deseription n'a pas été publiée, figure dans la collection du Muséum, récoltée sur Dicaeum sp. ? d'Indochine.

A. tridentatus Haller a été récolté par nous sur un pipit, Anthus richardi rufulus Vieillot, capturé à N'ha Trang en juillét 1946.

Genre Megninia (pattes de la troisième paire hypertrophiées chez le mâle, mais pourvues d'ambulacres comme celles des autres paires).

M. apodemata n. sp. (pl. II $a$ ).

Espèce à fente interlobaire partiellement comblée par une membrane transparente, proche parente de $M$. leucacantha, mais le tarse des pattes de la troisième paire ne porte que deux aiguillons transparents (fig. 2).

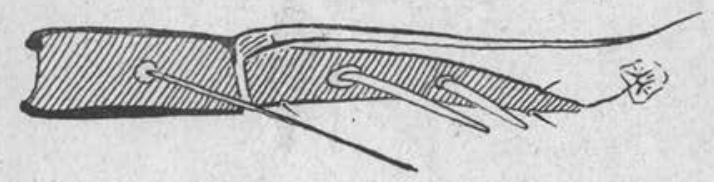

Fig, 2. - Extrémité d'une patte de la III' paire chez Megninia apodemata $\sigma^{\circ}$.

Les apodèmes, longuement étendus entre l'are génital mâle et les ventouses copulatrices, sont plus nettement visibles qu'il n'est habituel dans le genre. Epimères de la première paire de pattes contigus mais non confluents. Taille : mâle $0 \mathrm{~mm}$, 45 ; femelle $0 \mathrm{~mm}$., 35 .

Hòte: Dicrur us leucophrus mouhoti Walden.

Localité : Djiring, Sud-Annam (juin 1946).

M. columbær Buchholz a été régulièrement trouvé sur la tourterelle tigrine Streptopelia chinensis tigrina en Cochinchine.

M. ginglymura Megnin figure dans la collection Trouessart sous le nom d'Analges ginglymurus, réeolté sur Diardigallus diardi de Cochinchine.

Genre Mesalges (semblable à Megninia, mais le tarse des pattes de la quatrième paire tronqué ou atrophié chez le mâle).

$M$. diaphanoxus Bonnet a été trouvé en petit nombre sur Corvus macrorhynchus (Ban Me Thuot, février 1946).

M. elephantopus Trouessart figure dans la collection du Muséum, recueilli sur Dichoceros bicornis de Cochinchine.

M. oscinum Trouessart, parasite cosmopolite et ubiquiste, a été récolté, toujours en faible densité, chez divers moineaux : Passer montanus malaccensis Dubois et Passer flaveolus Blyth de Cochinchine. 
Proctophyllodinæ. - Dans cette sous-famille, les pattes antérieures sont inermes. L'abdomen est bilobé chez les deux sexes. Les deux lobes portent généralement des ornements chitineux (poils gladiformes, feuilles transparentes, etc...).

Genre Alloptes (les pattes de la quatrième paire sont hypertrophiées chez le mâle).

C'est à A. crassipes Canestrini que nous croyons devoir rapporter l'espèce rencontrée sur une bécassine Capella stenura Bonaparte, tuée en Cochinchine.

Genre Proctophyllodes (les lobes abdominaux du mâle terminés

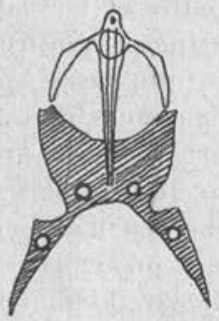

Fig. 3. - Organe génital mâle et apodème du Proctophyllodes de Passer montanus.

chacun par une feuille transparente. Les lobes abdominaux de la femelle portant des longs poils gladiformes).

Chez Passer montanus malaccensis Blyth, nous avons récolté en assez grand nombre une espèce voisine de $P$. profusus (pl. II $e$ ). Nous avons dit ailleurs (1948) que le groupe $P$. profusus- $P$. ampelidis devait être révisé et nous avons montré l'intérêt qu'il y aurait à utiliser pour cette révision les caractères fournis par la forme des apodèmes. En attendant et pour ne pas compliquer inutilement la synonymie du groupe, nous nous bornons à donner une figure des apodèmes de l'espèce rencontrée sur Passer montanus (fig. 3).

Genre Pterodectes (espèces allongées. Chez la femelle, les extrémités de l'arc chitineux vulvaire se raccordent aux épimères des pattes postérieures, l'ensemble de cette architecture chitineuse figurant un arc mauresque. L'extrémité des lobes abdominaux du mâle porte des ornements variés).

L'identification des espèces de ce genre recueillies sur les oiseaux d'Indochine nous a coûté un gros travail et reste douteuse.

$P$. lanceolatus? - Sugimoto (1942) a décrit, sur Lonchura (= munia) atracapilla de Formose, un Pterolichus lanceolatus. La 
description, comme les figures, montre qu'il s'agit d'un Pterodectes. Nous voyons peu de différences entre les figures de Sugimoto et les acariens recueillis par nous, en petit nombre, sur Munia punctatula topela Swinhoè en Cochinchine.

$P$. mainati Trouessart, espèce facilement reconnaissable aux pattes de la première paire, curieusement dilatées chez la femelle (pl. II b), a été trouvé, toujours en faible densité, sur Acridotheres tristis et sur Gracupica leucocephala du Cambodge, de la Cochinchine et du Sud-Annam.

$P$. papillo n. sp. (pl. II $c$ et $d$ ).

Cette espèce, relativement petite et très allongée, est remarquable par le développement des arcs chitineux renforçant les côtés de la face ventrale. Grands, larges, fortement chitinisés, ils tranchent par leur couleur sombre sur le fond pâle de téguments plutôt minces. Avec les épimères, fortement dessinés aussi, ces arcs figurent grossièrement un papillon. Les antennes sont représentées par les épimères I, les ailes antérieures par les épimères II et les ares antérieurs. Les arcs postérieurs et les épimères III et IV figurent les ailes postérieures, prolongées en pointes. Un poil long et un piquant en lame de poignard sur les flancs dans les deux sexes. La plaque notogastrique des femelles est très étroite. Sa largeur n'atteint pas le tiers de la largeur du corps.

Chez le mâle, les flancs se continuent insensiblement en arrière avec les bords extérieurs de deux lobes abdominaux courts, séparés par une échancrure médiane en V renversé. Trois poils ornent chacun de ces lobes. L'externe est le plus petit, le médian le plus long. Les ventouses copulatrices sont relativement éloignées de l'échancrure abdominale. Le pénis, en forme de glaive allongé, dépasse les ventouses et atteint l'extrémité des lobes abdominaux.

Longueur : mâle $0 \mathrm{~mm}$., 40 ; femelle $0 \mathrm{~mm}$., 50 .

Hôte : Ploceella chrysea Hume (tisserin doré), Basse-Cochinchine.

Une espèce voisine de $P$. mainati, mais aux pattes antérieures non dilatées chez la femelle, a été recueillie sur Corvus macrorhynchus (Ban Me Thuot, février 1946). Nous croyons devoir rapporter cette espèce à $P$. phyllurus décrit par Trouessart sur Manucodia atra de Nouvelle-Guinée. L'unique mâle que nous possédions diffère cependant de la description de Trouessart par la forme pointue de la feuille abdominale et par l'absence d'apodèmes. Il s'agirait donc d'une variété nouvelle: $P$. phyllurus oxyphyllus.

Genre Trouessartia (l'abdomen du mâle, très atténué en arrière, se termine par deux lobes étroits, ornés d'appendices transparents en nageoires de poisson, en roue dentée, etc...). 
T. eulabis Trouessart a été trouvé en grande abondance chez trois corbeaux Corvus macrorhynchus tués à Ban Me Thuot.

T. eulobulata Trouessart est un parasite habituel et abondant de Munia punctatula en Cochinchine. C'est cet acarien que Sugimoto $(4 b)$ décrit sur Lonchura (= munia) atracapilla de Formose comme une espèce nouvelle: Alloptes lonchuræ.

T. grandis Trouessart. Deux exemplaires seulement de cette grande espèce ont été recueillis sur Munia punctatula de Cochinchine.

T. marginata Trouessart a été trouvé abondant sur Acridotheres tristis et Gracupica leucocephala en Cochinchine, Sud-Annam et Cambodge (pl. I $c$ et $d$ ).

T. minuscula Trouessart a été rencontré sur le tisserin doré Ploceella chrysea de Cochinchine.

\section{Catalogue des espèces identifiées existant certainement en Indochine}

Pterolichinæ. - 11 espèces :

Falculifer rostratus.

Freyana leclerci.

Pseudalloptes calcaratus.

Pterolichus delibatus.

P. obtusus.

P. phylloproctus.

Analgesinæ. - 10 espèces :

Analges humulus.

A. tridentatus.

Megninia apodemata.

M. columbæ.

$M$. ginglymura.

Proctophyllodinæ. - 11 espèces :

Alloptes crassipes.

Proctophyllodes profusus.

Pterodectes lanceolatus.

$P$. mainati.

P. papillo.

P. phyllurus oxyphyllus.
P. rehbergi.

$P$. vexillarius.

Pteronyssus lagopus.

P. Ionchuræ.

P. truncatus.
M. ibidis.

Mesalges diaphanoxus.

$M$. elephantopus.

M. oscinum.

Psoroptoides psoroptopus.

A ces 32 espèces, on peut ajouter, sans grandes chances d'erreurs, les 22 espèces ci-après récoltées hors de l'Indochine, mais sur des oiseaux faisant partie de l'avifaune indochinoise. 
Pterolichinæ.

Freyana gracilipes Megnin et Trouessart sur Antigone sharpii (Chine).

F. tarandus Trouessart sur. Threskiornis melanocephalus (Indes).

Pterolichus amaurornis Sugimoto sur Amaurornis phænicura (Formose).

P. ornatus Megnin et Trouessart sur Paleornis torquatus (Indes).

$P$. parræ Megnin et Trouessart sur Hydrophasianus chirurgus (Indes).

P. subtruncatus Trouessart sur Gracula religiosa (Malaisie).

Pteronyssus nanus (1) Trouessart sur Ethopyga latouchei (Chine).

ANALgESIN Æ.

Hemialges humerosus Trouessart sur Eurylaimus javanicus (Malaisie).

H. tumens Trouessart sur Psarisomus dalhousiæ (Bornéo).

Megninia gallinulæ Buchholz sur Amaurornis phænicura (Formose).

M. ogivalis Trouessart sur Sula sula (Philippines).

Mesalges orientalis Trouessart sur Anthreptes malaccensis (Philippines).

M. pici majoris Buchholz sur Chelidorhynx hypoxanthum (Indes).

M. similis Bonnet sur Garrulax bellangeri (Bornéo).

Pteralloptes bipartitus Trouessart sur Anthracoceros malayanus (Insulinde).

P. corrugatus Trouessart sur Anthracoceros malayanus (Malaisie).

P. lyrurus Trouessart sur Hemichelidon sibirica (Chine).

\section{PROCTOPHYLLODINE.}

Allanalges gracilipes Trouessart sur Psarisomus dalhousiæ et Cymbirhynchus macrorhynchus (Malaisie).

Proctophyllodes pennifer Trouessart et Neumann sur Notodela leucura (Himalaya).

P. vegetans Trouessart sur Carpodacus erythrinus (Chine).

Pterodectes xiphiurus Trouessart sur Psarisomus dalhousiæ (Malaisie).

Trouessartia corvina Koch sur Enicurus leschenaulti (Chine).

\section{Catalogue par hôtes}

Nous réunissons les 54 espèces d'Analgesidæ dont la présence en Indochine est certaine ou probable dans le même tableau ci-après, établi en fonction de la position systématique des hôtes.

Pelecaniformes.

Sula sula $\ldots \ldots \ldots \ldots \ldots \ldots \ldots \ldots$ Megninia ogivalis.

Ardeiformes.

Threskiornis melanocephalus ..... Freyana tarandus.

(1) La description de cette espèce n'a pas été publiée, à notre connaissance. 
ANNALES DE PARASITOLOGIE T. XXIII, Nos 5-6, 1948.
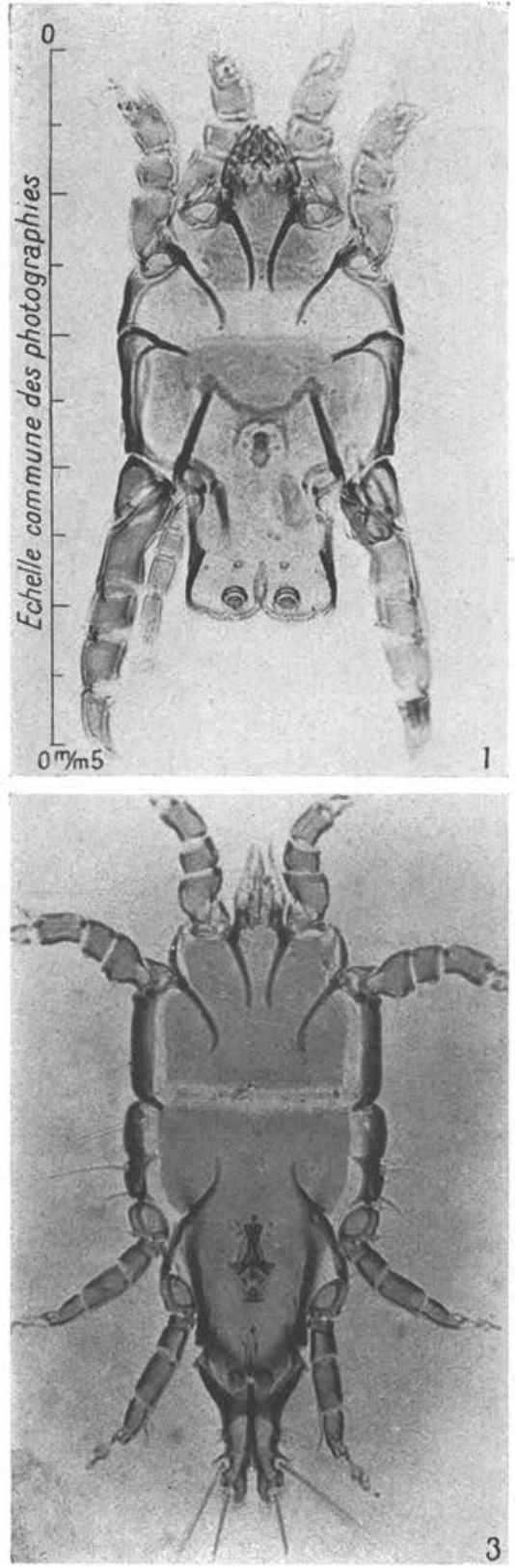

Planche X

(Mémoire Gavd el Petitot)
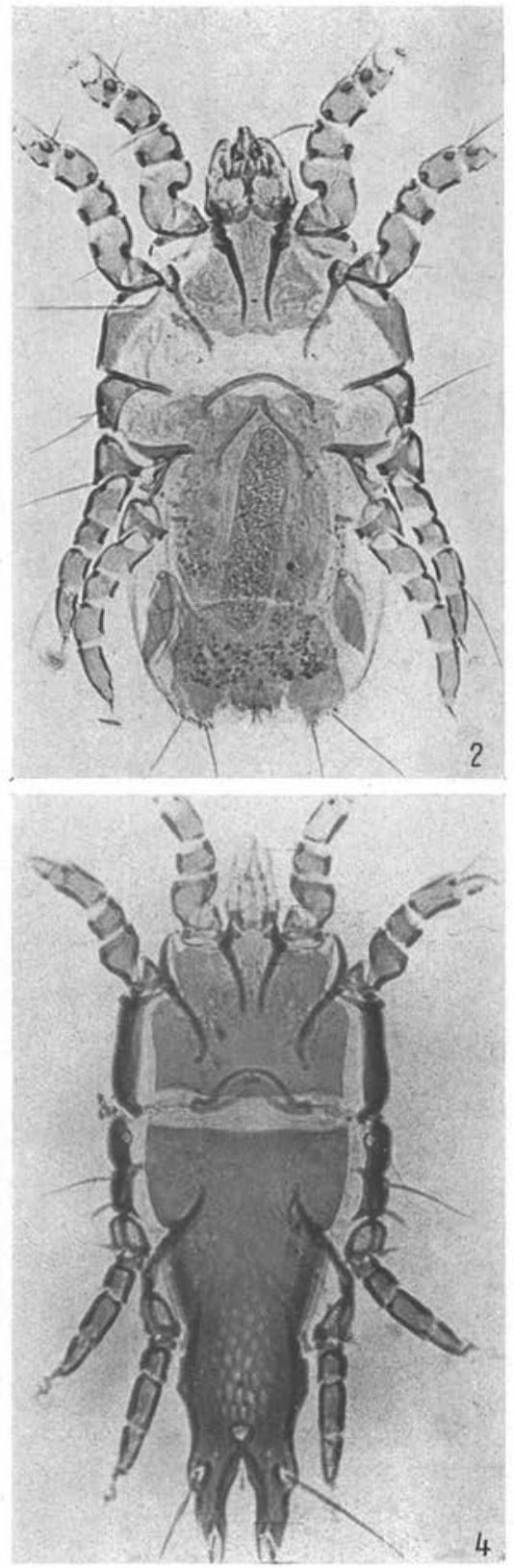

Planche $\mathrm{X}$

1. Pteronyssus truncatus $\delta$. 2. ㅇ. .

3. Trouessartia marginata oै. 4. 


\section{Plaxche XI}

5. Megninia apodemata oे.

6. Pterodectes mainati ㅇ.

7. $\quad P$. papillo .

8. idem $\delta$.

sur Ploceella chrysea

9. Proctophyllodes profusus?

de Passer montanus. 
ANNALES DE PARASITOLOGIE

T. XXIII, Nos 5-6, 1948.
Planche XI

(Mémoire Gaud et Petitot)
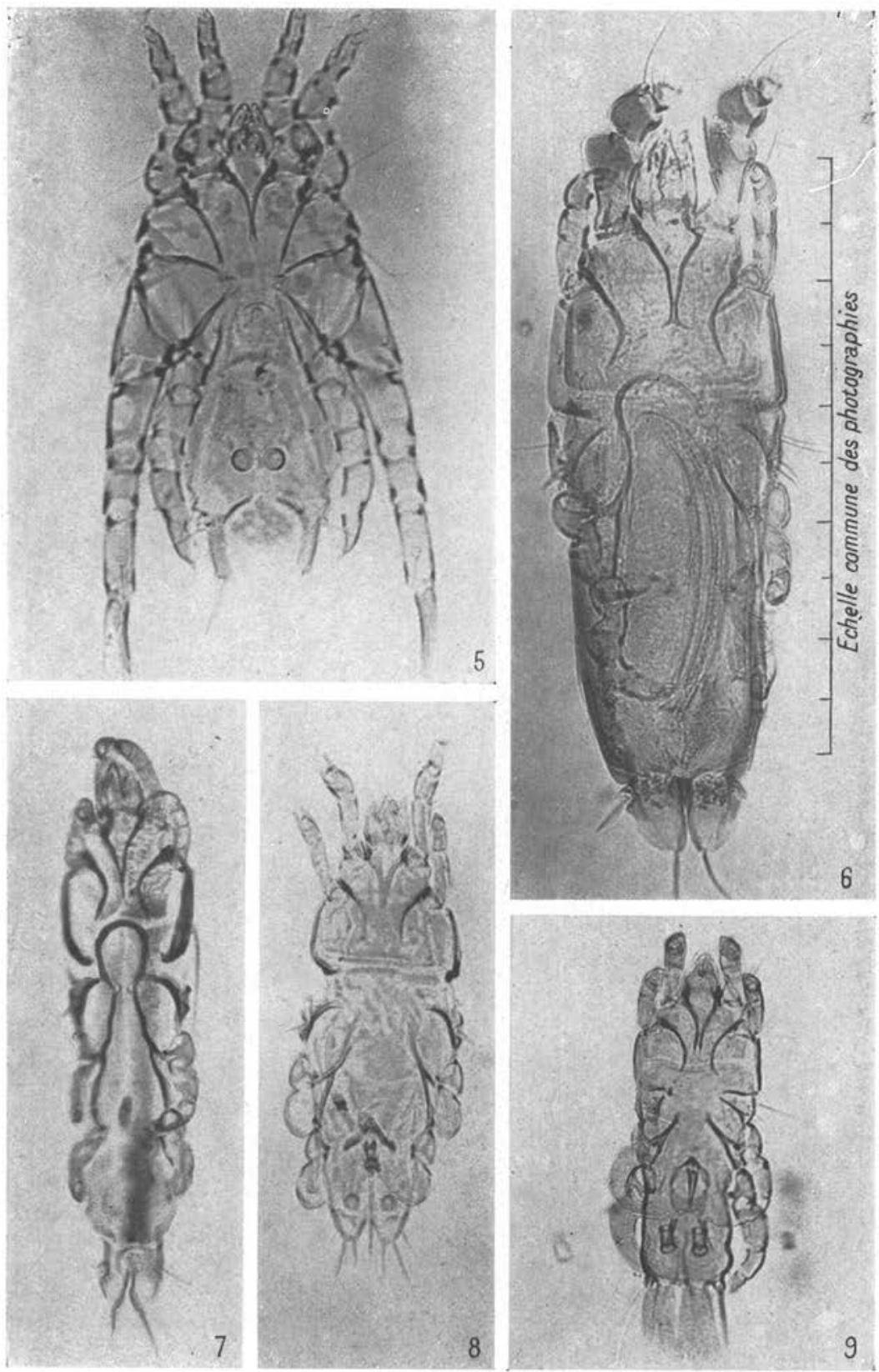

Masson et Cie, Editeurs 



\section{Charadrifformes.}

Capella stenura ............. Alloptes crassipes.

Lobivanellus indicus

Freyana leclerci.

Pterolichus rehbergi.

Gruiformes.

Antigone sharpii ................ Freyana gracilipes.

Hydrophasianus chirurgus ........ Pterolichus parræ.

Amaurornis phænicura ......... $\left\{\begin{array}{l}\text { Pterolichus amaurornis. } \\ \text { Megninia gallinulæ. }\end{array}\right.$

\section{Galliformes.}

Diardigallus diardi ........... $\left\{\begin{array}{l}\text { Pterolichus obtusus. } \\ \text { Megninia ginglymura. }\end{array}\right.$

Phasianus torquatus .......... Megninia ibidis.

Columbiformes.

Streptopelia chinensis $\ldots \ldots \ldots \ldots\left\{\begin{array}{l}\text { Falculifer rostratus. } \\ \text { Megninia columbx. }\end{array}\right.$

ACCIPITRIFORMES.

Haliëtus leucogaster ............ Plerolichus phylloproctus.

PsitTaciformes.

Palaornis torquatus ........... Pterolichus ornatus.

Coraciformes.

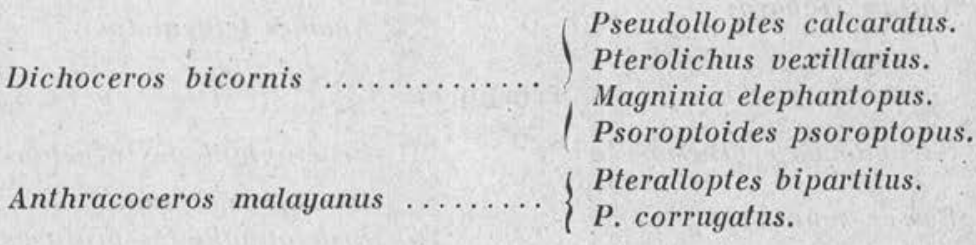

PASSERIformes.

Eurylaimidæ

Eurylaimus javanicus ............ Hemialges humerosus.

Cymbirhynchus macrorhynchus ... Pseudalloptes $s p$.

Psarisomus dalhousia $\ldots \ldots \ldots \ldots \ldots\left\{\begin{array}{l}\begin{array}{l}\text { Allanalges gracilipes. } \\ \text { Hemialges tumens. } \\ \text { Pterodectes xiphiurus. }\end{array} \\ \text { Pten }\end{array}\right.$

Muscicapidæ

Hemichelidon sibirica .......... Pteralloptes lyrurus.

Chelidorhynx hypoxanthum ...... Mesalges pici-majoris. 
Turdidæ

Enicurus leschenaulti .......... Trouessartia corvina.

Notodela leucura ............. Proctophyllodes pennifer.

Timaliidæ

Garrulax bellangeri ............ $\left\{\begin{array}{l}\text { Pteronyssus lagopus. } \\ \text { Mesalges similis. }\end{array}\right.$

Pycnonotidæ

Iole olivacea

Pteronyssus sp.

Dicruridæ

Dicrurus macrocercus ............

Dicrurus leucophæus ...........

Trouessartia reticulifer ?

Megninia apodemata.

Nectariinæ

Etopyga latouchei

Pteronyssus nanus.

Anthreptes malaccensis

Mesalges orientalis.

Dicæidæ

Dicæum $s$

Analges humulus.

Motacillidæ

Anthus richardi

Pteronyssus sp.

Analges tridentatus.

\section{Fringillidæ}

Carpodacus erythrinus .......... Proctophyllodes vegetans.

Passer montanus ............. $\left\{\begin{array}{l}\text { Mesalges oscinum. } \\ \text { Proctophyllodes profusus ? }\end{array}\right.$

Passer flaveolus .............., , Mesalges oscinum.

Proctophyllodes profusus?

\section{Ploceidæ}

Munia punctatula $\ldots \ldots \ldots \ldots \ldots \ldots \ldots, \begin{aligned} & \begin{array}{l}\text { Pteronyssus lonchuræ. } \\ \text { Pterodectes lanceolatus. } \\ \text { Trouessartia eulobulata. } \\ \text { T. grandis. } \\ \text { Pteronyssus lonchuræ. }\end{array} \\ & \begin{array}{l}\text { Pterodectes papillo. } \\ \text { Trouessartia minuscula. }\end{array}\end{aligned}$




\section{Sturnidæ}

Acridotheres tristis $\ldots \ldots \ldots \ldots \ldots$
Gracupica leucocephala $\ldots \ldots \ldots \ldots$

Gracula religiosa ............. Pteronyssus subtruncatus.

Pteronyssus truncatus. Pterodectes mainati. Trouessartia marginala.

Gorvidæ

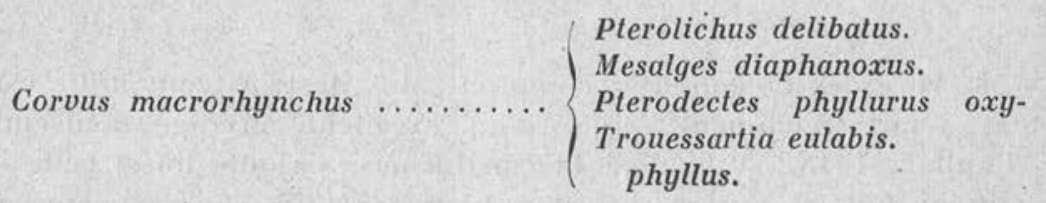

\section{BIBLIOGRAPHIE}

Bonnet (A.). - Révision des genres Megninia, Mesalges et genres voisins de la sous-famille des Sarcoptidés plumicoles. Bull. Soc. Zool, de France, XLIX, 1924.

Gavd (J.) et Petitot (M.-L.). - Sarcoptides plumicoles des oiseaux du Maroc. Ann. Parasit. hum, et comp., XXIII, 1948, p. 35-46, pl. I-II.

Houdemen (E.-F.). - Recherches de parasitologie comparée indochinoise. Paris, Le François, 1938.

Sucimoto (M.). - Studies on the Formosan mites. Bull. School of Agricult. Forestry Taiholu Imp. Univ., I. mars 1940 ; III, mars 1942.

Trouessart (E.). - Note sur la classification des Analgésiens. Bull. Soc, Et, Sc. Angers, XVI, 1886.

Révision des genres de la sous-famille des Analgesinæ ou sarcoptides plumicoles, Bull. Soc. Zool, de France, XL, 1915.

- et Megnin (P.). - Les sarcoptidés plumicoles. $J l$. de micrographie, VIII, 1884, et IX, 1885.

Institut d'hygiène de Rabat (Maroc). 